\title{
Cosmic-ray beryllium isotope ratio measured by BESS-Polar II
}

Takuya Wada ${ }^{a, \dagger, *}$ on behalf of the BESS Collaboration

(a complete list of authors can be found at the end of the proceedings)

${ }^{a}$ Institute of Space and Astronautical Science, Japan Aerospace Exploration Agency (ISAS/JAXA), Sagamihara, Kanagawa 252-5210, Japan

E-mail: twada@phys.aoyama.ac.jp

The Balloon-borne Experiment with a Superconducting Spectrometer (BESS) has carried out precise observation of the low-energy cosmic rays. Using a tracking system (in a $0.8 \mathrm{~T}$ uniform magnetic field) with a rigidity resolution of $\sim 0.4 \%$ at $1 \mathrm{GV}$ and a time-of-flight system with a time resolution of $120 \mathrm{ps}$, the BESS instrument enables us to distinguish isotope events. In December-January 2007-2008, BESS-Polar II achieved a 24.5-day observation during its balloon flight over Antarctica. By using 4.7 billion cosmic-ray event data obtained during the flight, rare ${ }^{10} \mathrm{Be}$ events have been searched and analyzed. In this paper, we will report the analysis process of the beryllium isotope ratio measured by the BESS-Polar II.

$37^{\text {th }}$ International Cosmic Ray Conference (ICRC 2021)

July 12 th - 23rd, 2021

Online - Berlin, Germany

\footnotetext{
${ }^{\dagger}$ Accepted in ISAS/JAXA under the cooperation agreement on the Cooperative Graduate School System between Aoyama Gakuin University and JAXA.

*Presenter
} 


\section{Introduction}

It has been almost 100 years since the first observation of galactic cosmic rays. The cosmic ray research has contributed to the discovery of new particles, and the development of high-energy physics that cannot be achieved with accelerators. Since the discovery of cosmic rays, the origin and propagation mechanism of these particles are major subjects for modern cosmic ray physics, and the nuclear components and their energy spectra in cosmic rays have been observed by many experiments. However, there are still no clear answers to these subjects.

As knowledge of cosmic ray propagation is essential for revealing the composition of the Galaxy, various propagation models and acceleration mechanisms have been proposed. A commonly used metric to constrain these models is the flux ratio of secondary to primary components among the cosmic radiation. The "primary" produced by sources such as carbon and oxygen interact with the interstellar material to produce "secondary" such as lithium, beryllium and boron. This ratio provides important information for understanding the propagation model, such as the average amount of interstellar material traversed by cosmic rays before they reach the Earth and the confinement time within the Galaxy. However, the ratio of stable particles such as $\mathrm{B} / \mathrm{C}$ cannot strongly constrain the confinement time. The most direct method to estimate the confinement time is to measure the abundance of radioactive components with a decay time comparable to the confinement time. Among them, the most attractive one is beryllium which has three isotopes $\left({ }^{7} \mathrm{Be},{ }^{9} \mathrm{Be}\right.$ and $\left.{ }^{10} \mathrm{Be}\right)$. ${ }^{10} \mathrm{Be}$ is unstable and has a decay time of $1.4 \mathrm{Myr}$ comparable to the average cosmic-ray confinement time within the Galaxy. Therefore, ${ }^{10} \mathrm{Be} /{ }^{9} \mathrm{Be}$ ratio provides strong constrains for the propagation models. The Be isotope ratio has not been reported, except for few cases such as ISOMAX[1], because of the small statistics of ${ }^{10} \mathrm{Be}$ and the difficulty of distinguishing these isotopes.

\section{The BESS Collaboration}

\subsection{The BESS Experiments}

The Balloon-borne Experiment with a Superconducting Spectrometer (BESS) is an international project to observe the low-energy cosmic rays, especially antiproton. The BESS has carried out precise observation of cosmic rays by using an instrument mainly consisting of a superconducting solenoid and a large tracker. By arranging a large tracker on a concentric axis in a thin superconducting solenoid, a larger geometrical acceptance and better momentum resolution were achieved. Since the first flight in 1993[2], BESS has successfully completed a total of 11 flights, including two experiments in Antarctica (The BESS-Polar I [3] and The BESS-Polar II[4]). For the flights over Antarctica, the BESS-Polar instrument[5][6] has inherited the features of the BESS instrument and has achieved a longer observation time and improved detector performance by fully developing the instrument, including reducing the materials used in the instrument. The second Antarctic flight in 2007-2008 (BESS-Polar II) achieved higher statistics and sufficient particle identification capability, and as a result, the BESS-Polar II data enabled us to identify beryllium isotopes. In this paper, we report the results of analysis using this data.

\subsection{The BESS-Polar II}

Figure 1 shows schematic cross-sectional and side views of the BESS-Polar II instrument. In a 


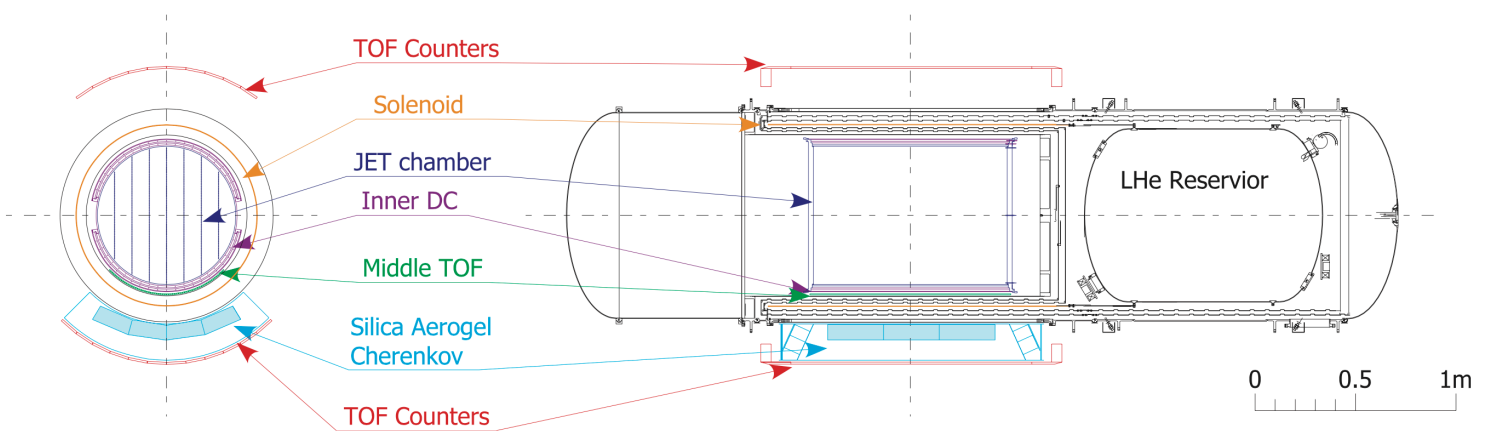

Figure 1: Cross-sectional and side views of the BESS-Polar II instrument.

uniform magnetic field of $0.8 \mathrm{~T}$ generated by a superconducting solenoid of $0.9 \mathrm{~m}$ diameter, a JETcell type drift (JET) chamber and Inner Drift Chambers (IDC) are arranged, and time-of-flight (TOF) counters and aerogel Cherenkov counter (ACC) are placed outside of them. The TOF counters, 10 in the upper (UTOF) and 12 in the lower (LTOF), measure the velocity $\beta(=v / c)$ of incident particles with a time resolution of $120 \mathrm{ps}$ and $\mathrm{d} E / \mathrm{d} x$ independently. The geometrical acceptance of $0.23 \mathrm{~m}^{2} \mathrm{sr}$ which is one order of magnitude higher than that of conventional balloon-borne detectors is achieved. The middle-TOF counters (MTOF) are installed under the lower IDC and on inner surface of the solenoid cryostat to measure low-energy particles that cannot pass through the lower solenoid. Incident particle track reconstruction is performed by fitting up to 52 tracker hits. The resolution of each point is $\sim 150 \mu \mathrm{m}$ in the $\mathrm{r}-\phi$ direction (perpendicular to the magnetic field) and $\sim 35 \mathrm{~mm}$ in the $\mathrm{z}$ direction (parallel to the magnetic field) for JET, and $\sim 140 \mu \mathrm{m}$ in the $\mathrm{r}-\phi$ direction and $\sim 800 \mu \mathrm{m}$ in the $\mathrm{z}$ direction for IDC. As a result, a rigidity $(R \equiv p c / Z e$, momentum per charge) resolution of $0.4 \%$ is obtained at $1 \mathrm{GV}$ and the maximum detectable $R$ is $240 \mathrm{GV}$. JET chamber also provides $\mathrm{d} E / \mathrm{d} x$ information.

The BESS-Polar II was launched from William Field near US McMurdo Station on December 22,2007 , and observed cosmic rays for 24.5 days. The flight altitude was $34-38 \mathrm{~km}$ (residual air of $5.8 \mathrm{~g} / \mathrm{cm}^{2}$ on average) and the cutoff rigidity was less than $0.5 \mathrm{GV}$. About 4.7 billion cosmic ray events were recorded.

\section{Data Analysis}

Because the BESS-Polar II instrument mainly uses track information of TOFs and JET chamber to identify particles, events in which multiple particles are injected into the instrument at the same time and interacted in the instrument cannot be accurately identified particles. In order to reject these events, only events with one hit each for UTOF and LTOF and one reconstructed track for JET were selected. In addition, track quality selections were applied, including fiducial cuts and quality cuts to obtain appropriate measurements.

In the BESS experiments, incident particles are identified by mass reconstruction using the following equation,

$$
M^{2}=(Z e R)^{2}\left(\frac{1}{\beta_{\mathrm{UL}}^{2}}-1\right) .
$$


The rigidity, $R$, is measured by the reconstructed particle track, and the velocity, $\beta_{\mathrm{UL}}$, is derived from the time-of-flight and the path length between the UTOF and LTOF. The charge, $Z$, is obtained from $\mathrm{d} E / \mathrm{d} x$ measured by the TOF and the JET chamber. Figures $2 \mathrm{a}$ and $2 \mathrm{~b}$ show scatter plots of $\mathrm{d} E / \mathrm{d} x$ measured by UTOF and LTOF against $R$. Figures $2 \mathrm{c}$ and $2 \mathrm{~d}$ show scatter plots of $\mathrm{d} E / \mathrm{d} x$ that rejected proton and helium events to focus on the high-charge $(Z \geqq 3)$ events. These plots indicate that BESS-Polar II provides enough $\mathrm{d} E / \mathrm{d} x$ resolution to identify the high-charge events. By using these measurements, incident particles including Be events can be identified. For the Be isotopes analysis, we confirmed that the $\mathrm{d} E / \mathrm{d} x$, rigidity and timing resolution for the high-charge events were stable throughout the flight as well as proton/helium events.

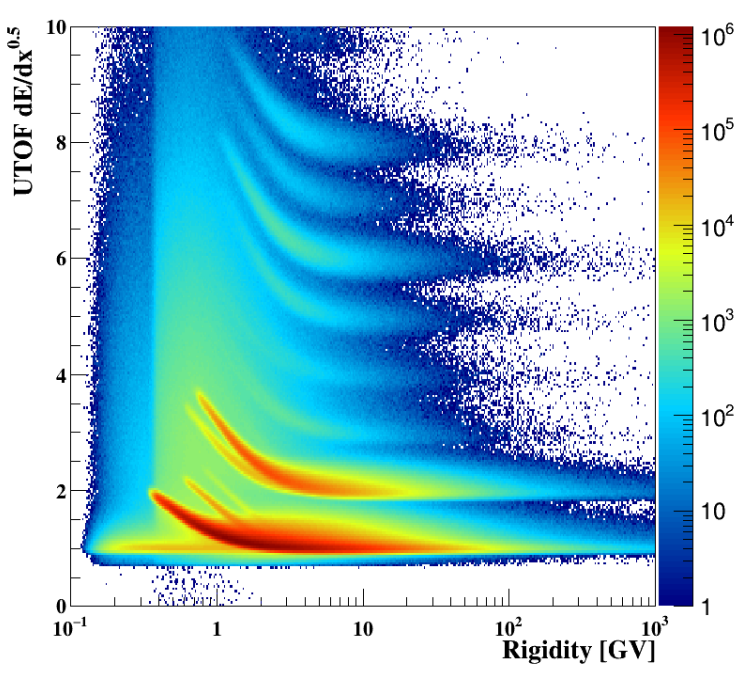

(a)

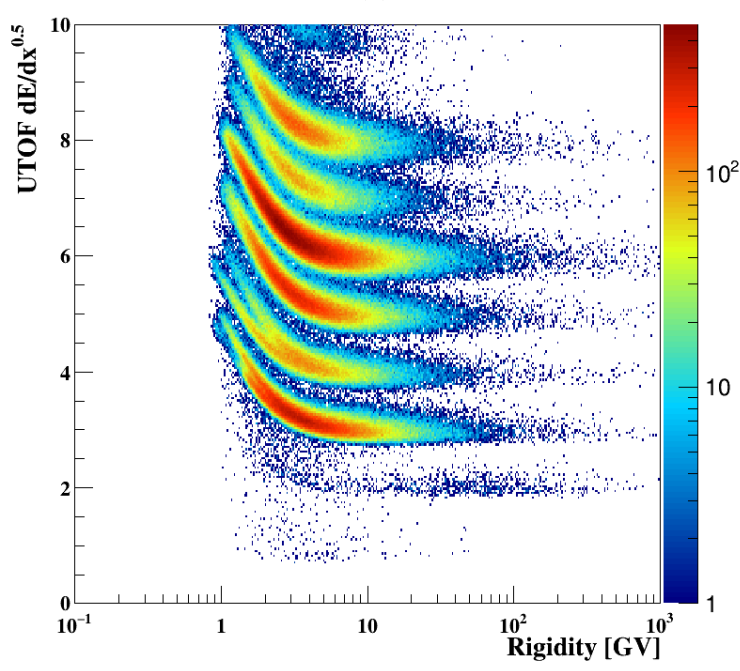

(c)

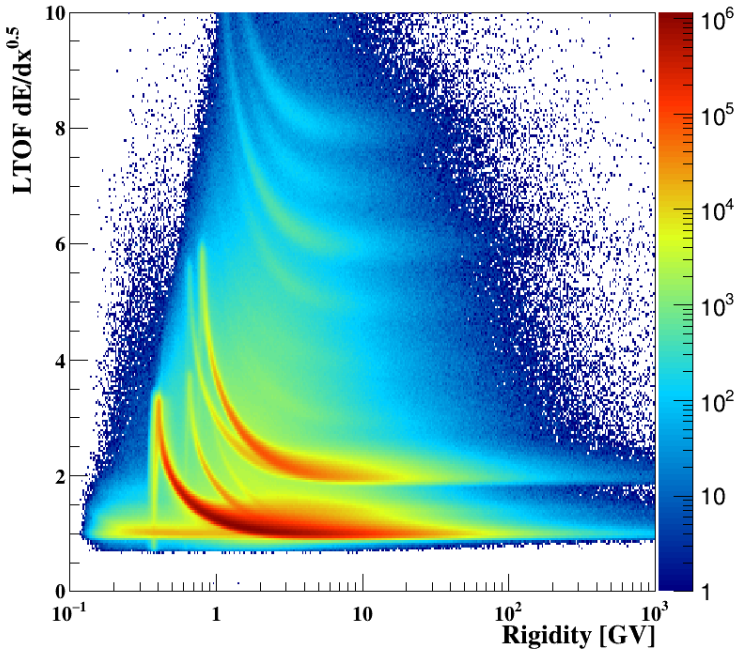

(b)

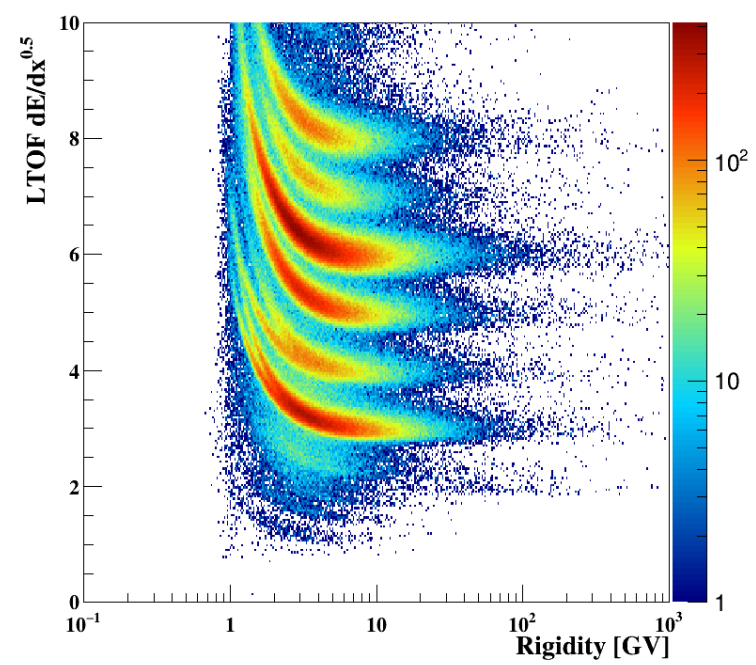

(d)

Figure 2: Scatter plots of $\mathrm{d} E / \mathrm{d} x$ from the (a)UTOF and (b)LTOF against the rigidity, $R$. To focus on the high-charge $(Z \geqq 3$ ) events, the $\mathrm{d} E / \mathrm{d} x$ distributions with eliminated proton and helium events are shown in (c) and (d).The high-charge events are well separated. 


\section{Data Selection for Be Events}

\section{1 $\mathrm{d} E / \mathrm{d} x$-band cut}

In order to select clean Be events, the $\mathrm{d} E / \mathrm{d} x$-band cuts for Be candidates are needed. Figures $3 \mathrm{a}-$ $3 c$ show scatter plots of $d E / d x$ with all cuts other than itself versus rigidity at the (a)UTOF, (b)LTOF and (c)JET chamber. For $\mathrm{d} E / \mathrm{d} x$ in JET chamber, a truncated mean method that the points in lower $10 \%$ and higher $55 \%$ are rejected and the mean $\mathrm{d} E / \mathrm{d} x$ is calculated using the rest of the hit points is adopted to remove the landau tail of the $\mathrm{d} E / \mathrm{d} x$ distribution and to prevent contamination of other particles. For these plots, $\mathrm{d} E / \mathrm{d} x$-band cuts are determined respectively to reject other elements events and are shown as solid black lines in Figure 3. By applying these $\mathrm{d} E / \mathrm{d} x$-band cuts, the Be candidate events were selected.

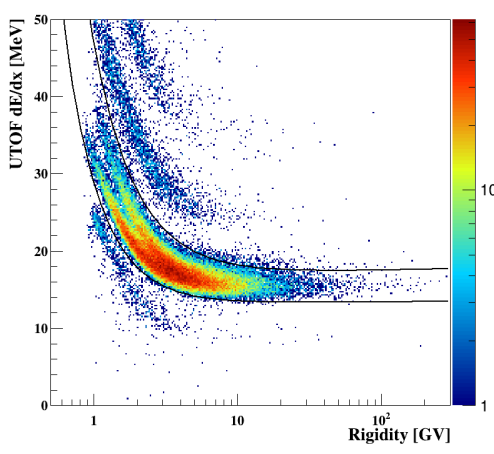

(a)

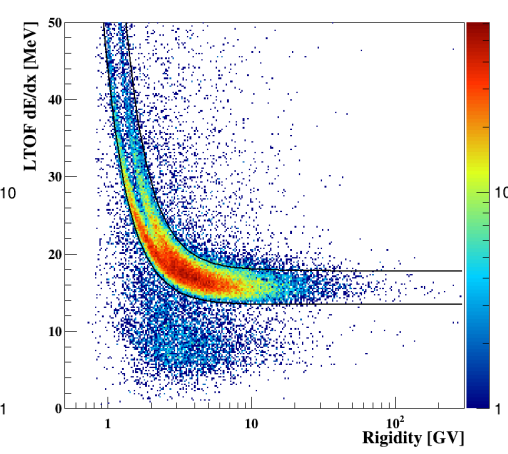

(b)

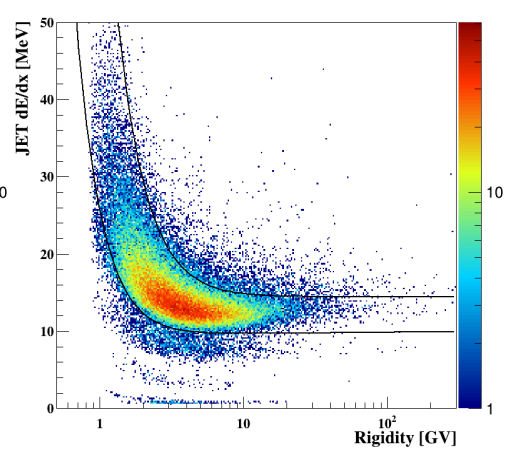

(c)

Figure 3: Scatter plots of $\mathrm{d} E / \mathrm{d} x$ from (a)UTOF, (b)LTOF and (c)JET with all cuts other than itself versus rigidity. The black solid line indicates $\mathrm{d} E / \mathrm{d} x$-band cut for Be candidates.

\subsection{Mass Distributions}

Figure 4 shows scatter plots of $1 / \beta_{\mathrm{UL}}$ versus rigidity under above $\mathrm{d} E / \mathrm{d} x$-band cuts applied. The theoretical lines of $1 / \beta_{\mathrm{UL}}$ for Be isotopes are shown as the black $\left({ }^{7} \mathrm{Be}\right)$, red $\left({ }^{9} \mathrm{Be}\right)$ and blue $\left({ }^{10} \mathrm{Be}\right)$ solid lines in Figure 4 . The Be candidates extracted by all the cuts are in good agreement with the theoretical lines of $1 / \beta_{\mathrm{UL}}$, indicating that the correct Be events selection is achieved. However, the contamination of other elements for the selected Be candidates is unavoidable. It is necessary to estimate these effects through detailed study.

The mass is calculated by Eq.(1) using $1 / \beta_{\mathrm{UL}}$ and $R$ in Figure 4 . Since $R$ is the rigidity obtained from the JET chamber of the center, conversion $R$ to kinetic energy at top of the instrument (TOI) is needed. The kinetic energy at TOI is calculated by correcting for the energy deposits in the instrument for the assumed particle species. The distribution of the mass and kinetic energy at TOI is divided into several energy region, perform fitting for the mass histograms, and the ${ }^{10} \mathrm{Be} /{ }^{9} \mathrm{Be}$ ratios at TOI are calculated in each energy region.

\section{3 ${ }^{10} \mathrm{Be} /{ }^{9} \mathrm{Be}$ ratio at the top of atmosphere}

The resulting the ${ }^{10} \mathrm{Be} /{ }^{9} \mathrm{Be}$ ratios at TOI include the effects of the residual atmosphere from space to TOI. In order to remove these effects and calculate the ${ }^{10} \mathrm{Be} /{ }^{9} \mathrm{Be}$ ratio at $\mathrm{TOA}$, conversion 


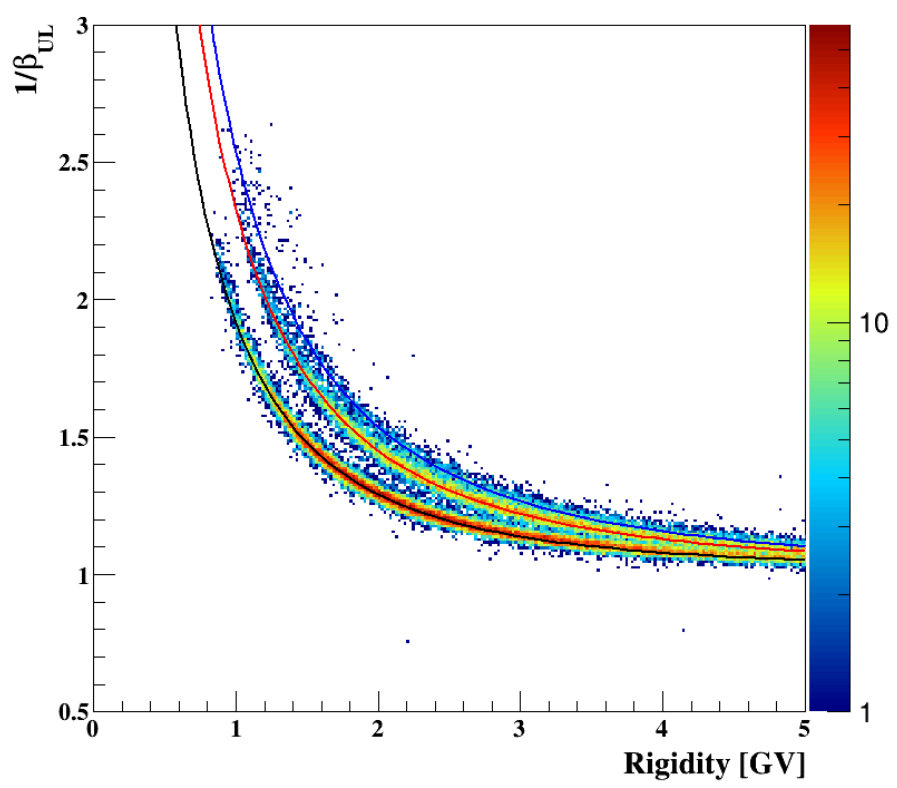

Figure 4: Scatter plots of $1 / \beta_{\mathrm{UL}}$ versus rigidity under $\mathrm{d} E / \mathrm{d} x$-band cuts applied. The black $\left({ }^{7} \mathrm{Be}\right)$, red $\left({ }^{9} \mathrm{Be}\right)$ and blue $\left({ }^{10} \mathrm{Be}\right)$ solid lines indicate $1 / \beta_{\mathrm{UL}}$ theoretical equation Eq.(1).

from the isotope ratio at TOI to value that takes into account the effect of the residual atmosphere is needed. The residual atmospheric effects are estimated by performing atmospheric simulation of Monte Carlo with data that local interstellar spectra and solar modulation at the same time as the flight period of BESS-Polar II obtained from GALPROP[7][8]. The propagation analysis enable us to derive an atmospheric correction for the Be isotope ratio.

\section{Summary}

We have demonstrated that the BESS-Polar II has superior instrument performance to identify the high-charge events and enough statistics to calculate the Be isotope ratios. Since the analysis method for determining the number of Be isotope events in the BESS-Polar II has been established, the detailed ${ }^{10} \mathrm{Be} /{ }^{9} \mathrm{Be}$ ratios are under careful evaluation following to the method.

\section{References}

[1] T. Hams, et al., ApJ 611, 2, 892-905 (2004).

[2] A. Yamamoto, et al., Adv. Space Res. 14, 2, 75-87 (1994).

[3] K. Abe, et al., Phys. Lett. B, 670, 2, 103-108 (2008).

[4] K. Abe, et al., Phys. Rev. Lett., 108, 5, 051102 (2012).

[5] A. Yamamoto, et al., Adv. Space Res. 30, 5, 1253-1262 (2002).

[6] J. W. Mitchell, et al., Nucl. Phys. (Proc. Suppl.) 134, 31-38 (2004) 
[7] GALPROP Webrun https://galprop.stanford.edu/webrun.php

[8] A. E. Vladimirov, et al., Comp. Phys. Comm., 182, 5, 1156-1161 (2011) 


\section{Full Authors List: BESS Collaboration}

K. Abe ${ }^{1, \star}$, H. Fuke ${ }^{2}$, S. Haino ${ }^{3, \dagger}$, T. Hams ${ }^{4}$, M. Hasegawa ${ }^{3}$, K. C. Kim ${ }^{5}$, M. H. Lee ${ }^{5}$, Y. Makida3, J. W. Mitchell ${ }^{4}$, J. Nishimura ${ }^{6}$, M. Nozaki ${ }^{3}$, R. Orito ${ }^{1, \pitchfork}$, J. F. Ormes ${ }^{7}$, K. Sakai ${ }^{4}$, M. Sasaki ${ }^{4}$, E. S. Seo ${ }^{5}$, R. E. Streitmatter ${ }^{4}{ }^{\S}$, N. Thakur ${ }^{4}$, T. Wada $^{2}$, A. Yamamoto ${ }^{3}$, T. Yoshida ${ }^{2}$ and K. Yoshimura ${ }^{8}$

${ }^{1}$ Kobe University, Kobe, Hyogo 657-8501, Japan. ${ }^{2}$ Institute of Space and Astronautical Science, Japan Aerospace Exploration Agency (ISAS/JAXA), Sagamihara, Kanagawa 252-5210, Japan. ${ }^{3}$ High Energy Accelerator Research Organization (KEK), Tsukuba, Ibaraki 305-0801 Japan. ${ }^{4}$ NASA-Goddard Space Flight Center (NASA-GSFC), Greenbelt, MD 20771, USA. ${ }^{5}$ IPST, University of Maryland, College Park, MD 20742, USA. ${ }^{6}$ The University of Tokyo, Bunkyo, Tokyo 113-0033, Japan. ${ }^{7}$ University of Denver, Denver, CO 80208, USA. ${ }^{8}$ Okayama University, Okayama, Okayama 700-0082 Japan.

${ }^{\star}$ Present address: Kamioka Observatory, ICRR, The University of Tokyo, Hida, Gifu 506-1205, Japan

${ }^{\dagger}$ Present address: Institute of Physics, Academia Sinica, Nankang, Taipei 11529, Taiwan

‡Present address: Tokushima University, Tokushima, Tokushima 770-8502, Japan

$\S_{\text {deceased }}$ 